\title{
Blended learning y la convergencia entre la educación presencial y a distancia
}

\section{Blended learning and the convergence of face-to-face and distance education}

\author{
Lorenzo García Aretio \\ UNED (España)
}

\section{Resumen}

La convergencia, la confluencia, entre las metodologías y recursos de los sistemas educativos presenciales y a distancia está siendo hoy una realidad. Una de las variantes de las instituciones duales o mixtas es la de ofrecer un determinado programa, asignatura o curso con una combinación de tiempos cara a cara, en el aula, y otros tiempos de trabajo fuera del recinto académico, con el apoyo de las tecnologías. Se trataría del blended-learning. Aclaremos que estas mezclas y combinaciones de métodos y recursos, ya se venían realizando por parte de las primeras universidades a distancia con el apoyo de las tutorías presenciales, mucho antes de la llegada de las tecnologías digitales. En este trabajo nos inclinamos más que por mezcla o combinación de modalidades educativas, por la integración de medios, recursos, tecnologías, metodologías, actividades, estrategias y técnicas, tanto presenciales como a distancia, para satisfacer cada necesidad concreta de aprendizaje. Como una variante más de esta integración aparece la flipped classroom en la que se contemplan tiempos en el aula y en el hogar poniendo el énfasis en reorganizar el trabajo de los estudiantes en el aula (más interacción) y en su hogar (más trabajo autónomo), de forma inversa a como venía siendo habitual, así como el nuevo rol del profesor.

Palabras clave: educación a distancia; e-learning; convergencia de sistemas; blendedlearning; flipped-classroom.

\begin{abstract}
The convergence, the confluence, between the methodologies and resources of the faceto-face and distance education systems is now a reality. One of the variants of dual or mixed institutions is to offer a particular program, subject or course with a combination of face-toface moments in the classroom, and other work activities outside the academic institution with the support of technologies. It is the "blended-learning". We clarify that this blend and combination of methods and resources was already being carried out by the first distance universities with the support of face-to-face tutorials long before the arrival of digital technologies. In this work, rather than consider it a blend or combination of educational modalities, we are inclined to consider it as an integration of means, resources, technologies, methodologies, activities, strategies and, both face-to-face and distance learning, techniques to satisfy each specific learning need. As a further variant of this integration there appears
\end{abstract}


the "flipped classroom" in which there are times planned to be spent in the classroom and times to be at home with the emphasis placed on reorganizing the work of the students in the classroom (more interaction) and at home (more autonomous work), as opposed to what used to be, as well as the new role of the teacher.

Keywords: distance education; e-learning; systems convergence; blended-learning; flippedclassroom.

Entre las instituciones educativas o programas unimodales, de sólo educación a distancia, es posible que queden algunos que aún no cuenten con servicios virtuales. Se trataría de instituciones que continúan impartiendo una educación a distancia de corte convencional, dirigida fundamentalmente a sectores de la población con problemas de acceso a las tecnologías y a la conectividad. Este modelo está en extinción, aunque puede seguir siendo necesario para ese tipo de poblaciones existentes en países del mundo en vías de desarrollo (García Aretio, 2014). Esa educación a distancia basada en soportes texto impreso, teléfono, correo postal y radio puede continuar prestando un servicio inestimable en algunas zonas del globo cuyas condiciones orográficas, socioeconómicas o tecnológicas impiden implementar aún una educación a distancia soportada en sistemas digitales y de telecomunicación.

Obviamente, son más aquellas instituciones unimodales que habitualmente ofrecían todos sus programas íntegramente a distancia por procedimiento convencional y que progresivamente fueron incorporando los sistemas digitales en su quehacer, pero sin perder del todo el componente más convencional. Otras, bien porque nacieron así o bien porque progresivamente los han ido adaptando, todos sus servicios de formación los prestan a través de sistemas virtuales.

De aquellas instituciones que se han venido denominado duales, bimodales o mixtas (distancia-presencia), las más habituales hoy día, podríamos señalar que disponen de los dos modelos clásicos, el "presencial” que atiende a los estudiantes que acuden a sus aulas, bibliotecas y laboratorios, con la metodología habitual y el "a distancia", que implica que, dentro del mismo centro o institución, existen estudiantes que siguen los estudios, total o parcialmente, a través de esta modalidad. También en estos centros duales o bimodales pueden encontrarse casos en los que en unos determinados estudios se ofrecen materias o asignaturas en formato presencial y el resto plenamente a distancia (García Aretio, 2012).

\section{LA CONVERGENCIA}

En la sociedad actual, cada vez son menos las instituciones presenciales modernas que no cuentan en su docencia con el correspondiente complemento virtual. Además de las tradicionales clases y seminarios presenciales, se ponen a disposición de los estudiantes sitios Web de las asignaturas, plataforma digital, foros, chat, blogs, redes 
sociales, wikis, tareas, etc. Este submodelo viene siendo progresivamente introducido por buena parte de las instituciones ordinarias presenciales.

Algunos centros imparten a distancia ciertos estudios o algún programa o materias que también se siguen ofreciendo en modalidad presencial. Sin embargo, lo que ahora nos interesa en este trabajo, resulta más común encontrarnos con instituciones que imparten estudios con un determinado porcentaje de tiempo lectivo realizado en presencia y el resto a distancia. Este submodelo viene denominándose como de estudios semipresenciales o de blended-learning.

Ya hace 20 años, García Aretio y Marín Ibáñez (1998), apuntaban que las enseñanzas presencial y a distancia se ordenan sobre una línea continua, en uno de cuyos extremos estaría el momento en que el profesor, cara a cara con el alumno, dirige su aprendizaje. En el otro extremo se halla el estudio del alumno aislado, que recurre a un sistema multimedia y consulta las fuentes de un modo autónomo para adquirir los conocimientos, destrezas y actitudes, que estima válidos para elevar su calidad de vida. Pero, ni el sistema educativo presencial ni las enseñanzas a distancia, señalaban estos autores, cumplen íntegramente las exigencias que se agudizan en uno y otro extremo. Hay alumnos que necesitan la presencia de los profesores y los compañeros, pero hay quienes aprenden mejor en el silencio y la soledad. La diferencia, pues, entre la enseñanza presencial y a distancia es una cuestión de grado, no el salto radical entre el sí y el no, la permanente presencia o la ausencia total, el contacto vivo o la desoladora lejanía.

De ahí el que pudiéramos cuestionarnos, ¿dónde estaría hoy, pues, la frontera entre lo presencial y lo "a distancia"?, ¿no se está diluyendo cada vez más, o quizás ya esté diluida?, ¿podría una formación universitaria moderna reducirse exclusivamente al contacto profesor-alumno en el aula? Los sistemas a distancia, cierto que dependiendo del nivel de estudios, ¿̇pueden prescindir $100 \%$ de algún contacto presencial, aunque sea en algún momento de la evaluación en estudios oficiales y reglados?, ¿no sería mejor hablar de diferente énfasis que, según modelos, se asigna a la relación presencial y la soportada, por ejemplo, en entornos virtuales de aprendizaje?, ¿no es más cierto que cada vez existe menor salto entre unas y otras experiencias, modelos o formulaciones?, ¿̇no es más cierto que deberíamos hablar de confluencia o convergencia de sistemas?, ¿no es más cierto que más que contraponer sistemas deberíamos hablar de educación, de calidad de esa educación, sea con más énfasis presencial o a distancia? (García Aretio, 2004a).

\section{EL BLENDED LEARNING}

Que la referida convergencia actual pueda llevar a lo que desde hace años se viene denominando como blended-learning, no quiere decir que este b-learning esté naciendo ahora, en estos años de este siglo XXI en los que se tiende a converger (Graham, 2005; Turpo, 2010). Con la denominación de blended-learning, parecería que nos encontráramos ante un sistema revolucionario, absolutamente nuevo, que 
fuera a solucionar todos los problemas educativos y de formación de la sociedad actual. La verdad es que leyendo alguna literatura al respecto así podría considerarse, dado que son numerosas las investigaciones que resaltan su eficacia frente a los modelos "limpios" de e-learning o totalmente presenciales (Niekerk y Webb, 2016; Means, Toyama, Murphy, Bakia y Jones, 2009; Siemens, Gašević y Dawson, 2015; Cotrell y Robison, 2003; Singh, 2003; Gülbahar y Madran, 2009; Usta y Ozdemir, 2007; Vaughan y Garrison, 2005; Laumakis, Graham y Dzubian, 2009).

Con el blended-learning se trataría, por una parte, de superar los vicios y deficiencias que acumula la enseñanza presencial, y por otra, de solucionar el posible estancamiento del e-learning (Aiello y Cilia, 2004) remediando, también, las debilidades propias de los sistemas virtuales plenos. Sería así una suerte de complementariedad.

En traducción literal, con blended learning nos estaríamos refiriendo al "aprendizaje mezclado" (to blend = mezclar, combinar), ¿diríamos aprendizaje combinado, mixto, híbrido, amalgamado, anexado, entreverado, entretejido, convergente, integrado, dual, bimodal, semipresencial...? (Salinas, 1997; García Aretio y Marín Ibáñez, 1998; Bartolomé, 2004; García Aretio, 2004a; Llorente, 2009). Ahora nos interesa resaltar el llamativo convencimiento de tantos articulistas o "prácticos" que asumen el e-learning desde hace algunos años, y el blended-learning más recientemente, como si de enfoques surgidos casi por generación espontánea se tratase. Es más, vienen a contraponerlos a la "denostada" educación a distancia, como si ésta hubiera que entenderla anclada exclusivamente en el material impreso, el correo postal y el teléfono (García Aretio, 2004a).

Es evidente que el e-learning (enseñanza y aprendizaje digitales), supuso una variante de modernidad que vino a sustituir los materiales y vías de comunicación propios de la educación a distancia de décadas pasadas, por soportes y redes digitales. Es decir, se trataba de ofrecer una educación a distancia de hoy que debe procurar una mayor calidad tanto en los contenidos y su presentación, como en las interacciones simétricas, asimétricas, síncronas y asíncronas que, a través de las tecnologías digitales, se pueden generar. Según nuestra conceptualización amplia de educación a distancia, el e-learning, es educación a distancia, al basarse en un diálogo didáctico mediado entre el profesor (institución) y el estudiante que, ubicado en espacio diferente al de aquel, aprende de forma independiente y también colaborativa (García Aretio, 2001).

Así, aprovechando los principios pedagógicos más sólidos, la educación a distancia ha venido construyendo desde décadas atrás modelos institucionales/ organizativos, pedagógicos y tecnológicos que hoy pueden valer (que están valiendo) a las más serias propuestas (hay muchas que no son serias) de sistemas digitales de enseñanza y aprendizaje que se presentan en todo el mundo (García Aretio, 2004b).

Cuando desde la educación a distancia se establecen sesiones presenciales generalizadas, de carácter más o menos obligatorio, combinadas con tiempos propios de aquella educación a distancia de antes o del e-learning de hoy, o cuando desde la 
educación presencial se van recortando los tiempos "cara a cara" sustituyéndolos por la relación en espacios virtuales, surge la educación/enseñanza/aprendizaje semipresencial, mixta o combinada, el blended-learning. En estos casos se han querido recoger las ventajas de la buena educación a distancia, combinándolas con los probados beneficios de la buena formación presencial (Aiello y Cilia, 2004).

Las variantes de modelos con más o menos tiempos presenciales/distancia son infinitas, las posibilidades de combinación, inmensas. Pero ¿cuáles ofrecen mejores resultados? Nuestra experiencia y las numerosas investigaciones a las que nos hemos aproximado nos dictan que los resultados, la eficacia de estos cursos, programas y carreras dependen, no ya de la tecnología empleada y de la cantidad o proporción respectiva de presencia/distancia, sino de los diseños pedagógicos, de la metodología, del uso adecuado que se hace de los recursos y de la preparación y disposición del profesorado (García Aretio y Ruíz, 2010). Evidentemente, si:

- contamos con la tecnología requerida;

- el diseño es adecuado;

- aplicamos la metodología acomodada a la situación;

- ajustamos el uso que hagamos de los recursos;

- usamos con sentido pedagógico las tecnologías colaborativas;

- disponemos de unos equipos docentes, directivos y diseñadores bien capacitados, convencidos y altamente motivados;

si todo eso es así, los resultados positivos estarán garantizados, se ganará en eficacia y eficiencia. Aunque de nada sirve todo esto si los contenidos propios del curso o materia no son de calidad. Ya sabemos, puede escribirse un excelente libro con pluma de ave y tinta y un desastroso texto utilizando la última generación de procesadores.

Algunos modelos de educación a distancia, fueron evolucionando hacia el uso más sistemático de sesiones presenciales, aunque varios de ellos, posteriormente, dieron el giro contrario. Y desde la formación presencial, se viene evolucionando desde hace años, utilizando recursos que venían siendo más propios de una educación a distancia tecnológicamente más avanzada.

Como puede verse, las propuestas de blended learning no son tan recientes, aunque la denominación sí lo sea. Existen modelos que han venido utilizando estas combinaciones de secuencias presenciales y a distancia desde hace varios decenios (Bartolomé, 2004; García Aretio, 2004a). Han combinado adecuadamente diferentes recursos tecnológicos, más o menos convencionales, que han potenciado la relación presencial de los formatos clásicos. 


\section{¿POR QUÉ NACIÓ EL BLENDED LEARNING?}

Al blended learning, por tanto, bien está que a nivel de denominación lo consideráramos como nuevo, pero no así a nivel de innovación educativa radical y estructural. Textos, gráficos, voz, audio, animaciones, vídeo..., se vienen utilizando desde hace muchos años tanto en los formatos presenciales como en los propios de la educación a distancia más convencional. Cuando estas formas de comunicación didáctica fuimos capaces de plasmarlas, todas ellas, en soportes de multimedia digitales, e igualmente tuvimos la posibilidad de transmitir, recuperar y reproducir la información mediante las correspondientes redes digitales y los oportunos sistemas de codificación y descodificación, es cuando dimos el verdadero salto tanto cualitativo como cuantitativo en la comunicación educativa.

Entonces, ¿desde dónde nació el llamado blended learning? Pues, aunque pudiera parecer lo contrario, el blended learning ha sido una evolución, más que desde el e-learning, desde los procesos presenciales de formación en la empresa a los que después se sumaron instituciones educativas tradicionalmente presenciales, buena parte de ellas, universitarias. Tanto unos como otros, probablemente, al adoptar en su momento esta modalidad con ese nombre lo hicieron por alguna de estas razones (Bartolomé, 2004; García Aretio, 2009):

- Procurar, sinceramente, superar los vicios y deficiencias que acumulaba la enseñanza presencial, mediante el aporte de las tecnologías.

- Pretender un aggiornamiento introduciendo tecnologías, pero sin perder el "prestigio" alcanzado como institución presencial.

- Buscar el esnobismo, la moda, al introducir en sus productos de formación esta terminología en inglés. El marketing ante todo, por encima de otras consideraciones pedagógicas.

- Quedarse a medias al tratar de aprovechar los recursos educativos con que ya contaba la institución presencial.

- Optar por el previsible abaratamiento de costes.

- Apostar por un modelo mixto que les permitiera subsanar en "presencia" los fallos que pudieran cometerse en un proceso íntegramente en línea.

- Introducir suavemente la tecnología sin prescindir del "poder" de la relación presencial con lo que los formadores más opuestos pueden limitar su rechazo, porque están convencidos de que la relación presencial en educación es imprescindible.

En este caso de transición desde lo presencial a este modelo, parecería que el blended learning supone un paso adelante. Sin embargo, otros que miran desde el e-learning, piensan que se trata de un paso hacia atrás. Pero, curiosamente, también hay (sobre todo últimamente) quienes, desde la orilla del e-learning hablan sólo de paso adelante porque dicen, afortunadamente, se recuperó el protagonismo de la 
relación presencial y se resuelven vicios del e-learning (Bartolomé, 2004). Y resulta curioso que ahora los que venden blended learning, que antes vendían e-learning tratan de convencernos de los beneficios de la relación presencial entre seres sociales por naturaleza como son los alumnos y los docentes, a veces con argumentos que niegan las razones anteriores con las que defendían apasionadamente la enseñanza virtual $100 \%$. Hablan, incluso, de la recuperación del anhelado cara a cara (?). Los que antes se oponían radicalmente al e-learning, ahora nos destacan las ventajas de la incorporación (integración, diríamos) de las tecnologías en los procesos formativos. Éstos se han dado cuenta, aunque tarde, de que no se puede seguir cerrando la puerta de las instituciones de educación y formación a las tecnologías.

$\mathrm{Si}$ se entresacan las ventajas indudables que pueden aportar la enseñanza y aprendizaje íntegramente digitales, se seleccionan las mejores de ellas y se hace lo propio con las que reporta la relación presencial, ¿quién puede dudar de la interesante propuesta que surgirá? Sería mezclar lo mejor de ambos mundos (Reynolds y Greiner, 2005; Bersin, 2004). Sin embargo, esos beneficios suponemos que conllevan alguna pérdida o renuncia a ciertos principios consagrados en la enseñanza a distancia desde siempre como por ejemplo, la flexibilidad tempoespacial que, al menos en determinadas ocasiones, se vería dañada.

Dando un paso más, algunos lo vienen denominando como blended e-learning (Mantyla, 2001; Jen-Her, Robert, Tzyh-Lih, 2010). Quizás esta segunda designación se ajuste más a la sencilla definición que se viene aceptando del blended learning: forma de aprender que combina o mezcla los entornos presenciales con las tecnologías no presenciales (Coaten, 2003; Marsh, 2003; Bartolomé, 2004), ¿se trataría de un híbrido de ambas?, hybrid model. Parece que incluir la "e" de "electrónico" delimita el concepto. No se aceptaría en ese supuesto como blended learning, a la enseñanza semipresencial basada en modelos y tecnologías convencionales de educación a distancia. Los adalides de la denominación, ciertamente, no la contemplan.

Garrison y Vaughan (2008) afirman que el blended learning está en el centro de una evolución lógica y transformadora de la educación, sobre todo de nivel superior, y se basa en tres premisas fundamentales: reestructuración de los tiempos habituales de clase tradicional; integración de los tiempos de presencia y aprendizaje en línea y rediseño del curso para potenciar la participación de los estudiantes. Esta dinámica supone, según estos autores, una reconceptualización y reorganización de las propuestas de aprendizaje basadas en estas nuevas interacciones (García Aretio, 2014).

\section{ENSEÑANZA Y APRENDIZAJE INTEGRADOS}

Aunque muy conscientes de que el término en inglés se ha impuesto, blended learning, en lugar de hablar de "mezcla", nos inclinaríamos más por el término "integración", que nos llevaría a una denominación o idea semejante a la de "Modelo de enseñanza y aprendizaje integrados". Nos resistimos a eliminar términos que 
puedan inducirnos a ignorar la tarea del docente que, finalmente, es quien diseña y desarrolla el proceso de enseñanza. Ya sabemos, nadie lo duda, que el objeto final de cualquier acción formativa es el aprendizaje, pero lo que hay que diseñar, en lo que han de capacitarse los docentes es en las estrategias y técnicas para una enseñanza de calidad que deberían culminar en aprendizajes, presumiblemente, del mismo corte.

Desde las propuestas más rancias dentro del aula presencial, hasta los más innovadores modelos tecnológicos educativos de hoy, pasando por todas las generaciones de la educación a distancia, un altísimo porcentaje del éxito de acciones formativas estriba en la figura del docente, en el modelo pedagógico que éste asuma, en su formación, disposición, motivación y en la eficiente utilización de los medios tecnológicos adecuados para cada situación didáctica concreta (García Aretio y Ruíz Corbella, 2010).

Pero, vamos a lo de "blended". En esa denominación de "enseñanza y aprendizaje integrados", pretenderíamos asignarle al término "integrados" todo su amplio significado semántico, donde no falte ningún elemento ni parte del hecho educativoformativo, donde se da integridad plena al proceso. Se trataría así, no de buscar puntos intermedios, ni intersecciones entre los modelos presenciales y a distancia, sino de integrar, armonizar, complementar y conjugar los medios, recursos, tecnologías, metodologías, actividades, estrategias y técnicas..., más apropiados para satisfacer cada necesidad concreta de aprendizaje, tratando de encontrar el mejor equilibrio posible entre tales variables curriculares. Así, trataríamos de planificar cuidadosamente estas variables, con el fin de (Horn y Staker, 2011; García Aretio, 2009):

- Complementar las ventajas del aprendizaje presencial cara a cara con los contrastados beneficios de un aprender a distancia;

- Armonizar las ventajas del aprendizaje autónomo e independiente con las indudables de los aprendizajes colaborativos;

- Compensar adecuadamente las comunicaciones verticales o asimétricas con las horizontales o simétricas;

- Equilibrar en sus justas proporciones las comunicaciones síncronas, en directo, con las asíncronas o en diferido;

- Integrar las tecnologías más propias de la enseñanza presencial o de la más antigua enseñanza a distancia con las más sofisticadas, propias de los procesos asentados en tecnologías digitales;

- Combinar el uso de los materiales de estudio en los formatos más adecuados para cada situación concreta;

- Disponer las dosis necesarias de aprendizaje guiado en grupo con el aprendizaje en equipo y el de corte individual.

Como puede verse, no descubrimos nada nuevo. Determinadas propuestas radicadas desde la educación a distancia y otras, avanzadas, desde la propia 
formación presencial, han protagonizado intentos que recogían todos o parte de los puntos anteriores y que han dado sus frutos desde hace años.

A lo largo de los siglos, no han sido los sistemas educativos ejemplo alguno de evolución y progreso. Por el contrario, siempre se han destacado por su actitud conservadora. Sin embargo, en el siglo y medio que lleva de vida la modalidad a distancia, sí que ha dado muestras de adaptación a los nuevos tiempos y de integración sin reservas de las TIC en los procesos de formación. Por tanto, lo que deseamos destacar, no es que desde la educación a distancia se esté dando este paso (esta modalidad nunca dudó en avanzar), sino que desde los ambientes más conservadores de la educación presencial, se vayan aceptando estos postulados.

\section{FLIPPED CLASSROOM}

Otro giro más reciente se le viene dando a estos conceptos desde la denominación de flipped classroom. Lage, Platt, y Treglia (2000) acuñaron el modelo del flipped classroom o del aula "invertida". Aunque aquella primera expresión no se consolidó hasta que en 2007 Bergman y Sams (Driscoll, 2012), del Instituto de Colorado de USA, se unen para grabar contenidos de presentaciones en PowerPoint narradas y capturadas en vídeo. Su idea era la de facilitar así a los alumnos que no asistiesen a algunas clases la posibilidad de no perder parte de las enseñanzas. Comprobaron posteriormente que estas grabaciones eran seguidas por otros estudiantes diferentes de aquellos hipotéticos destinatarios. Las experiencias de Khan Academy (García Aretio, 2011) cuentan también con componentes que en numerosos casos, en muchas escuelas, han propiciado un modelo similar al de la clase flipped (invertida, volteada o inversa).

Se trataría, en consecuencia, de hacer en casa lo que se hace ahora en el aula y en el aula lo que se hace en casa. Es una forma muy simple de definir este concepto. Si las enseñanzas del docente, su exposición, sus presentaciones en el aula, sus orientaciones generales, sus recomendaciones de lecturas, etc., pueden quedar grabadas en un vídeo o en una presentación narrada PowerPoint, Prezi o similar, estos materiales pueden ser visionados, estudiados, por los alumnos en su propio domicilio, con la particularidad de que pueden "rebobinar" cuantas veces sean precisas si es que algún concepto o idea no quedaron suficientemente claros. Aunque el énfasis no está en los vídeos, el esfuerzo estaría en cómo utilizar mejor los tiempos en el aula, dado que se eliminó carga de estudio fuera de la misma (Bergmann y Sams, 2013).

De esta manera, las tradicionales actividades, ejercicios o tareas que se suelen marcar en las aulas como trabajos para realizar en el hogar, en este modelo se desarrollarían en el aula, con la interacción con los otros pares y con la ayuda, apoyo, orientación y facilitación del profesor. Es una forma real de llevar el auténtico protagonismo del proceso al estudiante, liberando horas de enseñanza directa 
del profesor con el fin de poder dedicarlas a la mentoría, guía y facilitación de los aprendizajes de aquellos (Tourón y Santiago, 2015).

Naturalmente la simplificación de este modelo nos llevaría a argumentar en su contra en algunos de sus aspectos. Pero esa es la simplificación. Aquí existe tras el modelo una filosofía, otra forma de hacer educación, un planteamiento diferente al de la enseñanza tradicional. Sería una forma de redefinir el tiempo de clase como un entorno centrado en el estudiante (Bergmann y Sams, 2013). Cómo mejor utilizar el tiempo en el aula sería la clave, pero para ello, se precisan otros tiempos de estudio y trabajo por parte del estudiante.

Quizás, se trataría de que habilidades y competencias menos complejas en la escalera de Bloom, como el conocer (memoria, recuerdo) y el comprender (entender, justificar) podrían desarrollarse sin la presencia directa del docente. Sin embargo, otras habilidades tales como las de usar lo aprendido en situaciones diferentes (aplicación), distinguir, diferenciar los componentes, elementos, principios, propiedades, funciones... (analizar), comprobar, valorar, juzgar, probar... (evaluar), generar, producir, construir, elaborar...(crear)..., podrían llevarse a cabo en colaboración con los iguales y atendiendo a las posibles orientaciones del docente en el aula (Bergmann y Sams, 2013).

¿Podríamos hablar quizás de un blended learning evolucionado? Así, el blended tomado desde la educación a distancia más tradicional, trataba de incorporar a la misma las tecnologías, manteniendo los clásicos encuentros presenciales. Como evolución desde el e-learning, se trataba de buscar los beneficios incuestionables de alguna relación presencial en los procesos de enseñanza y aprendizaje.

Es decir, que hablar de flipped classroom, concepto de 2000 o 2007, sería diferente a reflexionar sobre esa filosofía desde una perspectiva más amplia. ¿̇ué se hacía o debería hacerse en las primeras tutorías presenciales de instituciones como la UNED o la Open University? Aquellos estudiantes contaban con los materiales de estudio, inicialmente casi todos en formato impreso y posteriormente, además del impreso, en audio, vídeo y después en formatos digitales. Los alumnos debían estudiar esos materiales, debían escuchar, visionar, navegar..., por los contenidos diseñados para ello. Posteriormente, en las sesiones presenciales de tutoría, se ponía en común el material estudiado, se resolvían dudas, se podía realizar alguna sesión de trabajo en grupos y se daban directrices para el estudio posterior.

Hoy desde la responsabilidad de los docentes se señalan los contenidos de estudio que pueden aparecernos en diferentes formatos. Se trabajan esos materiales para esas dos etapas básicas de la taxonomía de Bloom, el conocimiento (memoria o recuerdo de lo esencial) y comprensión de lo estudiado. Posteriormente atendemos a las actividades, tareas o ejercicios propuestos con el fin de aplicar, analizar, evaluar, crear, etc. Y ello lo podemos desarrollar en trabajos individuales o colaborativos, bajo la atenta mirada, presencial o virtual, del profesor que orienta, anima, facilita, resuelve dudas, etc. Y no olvidemos las inmensas posibilidades que muestran los modelos de aprendizaje entre pares, muy utilizados en el flipped clasroom. 
Aquella educación a distancia de las décadas de los 70, 80 y 90 del siglo pasado con tutorías presenciales, ¿no tenían mucho de blended learning?, ¿y no tenían algo de flipped classroom?

Estamos convencidos que desde siempre la educación a distancia ha ido por delante y claramente, se ve, va ganando (García Aretio, 2009). Ha ido por delante porque siempre estuvo ávida para integrar en sus diseños las tecnologías según fueron generándose. El teléfono, el audio, la radio, el vídeo, la televisión e Internet han sido tecnologías que sucesivamente vino incorporando la educación a distancia $\mathrm{y}$, posteriormente, la presencial.

Así es que flipped classroom ¿en nuestras escuelas, colegios, institutos, universidades? nos parecerá excelente y será de calidad si se hace bien y de forma coordinada por parte de todos los docentes (Jeong, González y Cañada, 2016). Es sabido que aquello que pueda explicar un magnífico profesor o aquella imagen o proceso difícilmente asequible a la mayoría y que puede hacerse factible a través de la fotografía o el vídeo, ¿̇por qué no aprovecharlo en nuestras asignaturas y cursos? Muchas instituciones de educación a distancia lo vienen haciendo desde hace mucho tiempo. En todo caso, como sucede casi siempre en educación, no cualquier educador va a tener éxito en este modelo, al igual que seguirá habiendo estudiantes que preferirán los modelos convencionales de corte presencial. Pero no cabe duda que las tecnologías están potenciando estas posibilidades. Por una parte redefinen la actuación de docentes y estudiantes en el aula y por otra le dan un giro radical a las tareas y actividades externas al aula (Devaney, 2014).

\section{CONCLUSIONES}

Podemos concluir con que, por una parte, las fronteras entre formatos educativos presenciales y a distancia, se empezaron a diluir hace años y que actualmente se va tendiendo cada vez más a la convergencia y confluencia de sistemas.

Las instituciones bimodales, duales o mixtas de enseñanza nos han venido mostrando el camino con diferentes propuestas y modelos, siempre contemplando las dos modalidades educativas, los dos extremos del continuo, la enseñanza presencial y la a distancia. Uno de esos modelos queda configurado por la utilización de las dos metodologías en la misma disciplina, asignatura o curso, de forma complementaria. Hoy estaríamos hablando de combinar la enseñanza cara a cara en el aula con las posibilidades que ofrecen las tecnologías para continuar aprendiendo fuera del recinto académico. Esto sería blended-learning con sus múltiples variantes y tipologías.

Pero esa combinación o mezcla de métodos y recursos en realidad vienen estando presentes desde que nació la educación a distancia universitaria, sobre todo, a partir de las décadas de los años 60 y 70 del pasado siglo, que ya combinaba con la más estricta educación a distancia los tiempos dedicados a las tutorías presenciales. Hoy 
con las tecnologías digitales son más instituciones y programas tradicionalmente presenciales los que se van acercando al blended-learning o blended-e-learning.

Y la más reciente modalidad de aula invertida o volteada (flipped classroom) la podríamos integrar también en una especie de modelo dentro de la mezcla que sugiere el blended-learning. En todos los casos, parecería apropiado hablar de integración, de enseñanza y aprendizaje integrados. Se trataría así, no de buscar puntos intermedios, ni intersecciones entre los modelos presenciales y a distancia, sino de integrar, armonizar, complementar y conjugar los medios, recursos, tecnologías, metodologías, actividades, estrategias y técnicas..., más apropiados para satisfacer cada necesidad concreta de aprendizaje, tratando de encontrar el mejor equilibrio posible entre tales variables curriculares.

\section{REFERENCIAS BIBLIOGRÁFICAS}

Aiello, M., y Cilia, W. (2004). El Blended Learning como práctica transformadora. Pixel-Bit, 23, 21-26.

Bartolomé, A. R. (2004). Blended Learning. Conceptos básicos. Pixel-Bit, 23, 7-20.

Bergmann, J., y Sams, A. (2013). Flip Your Students' Learning. Educational Leadership, 7o(6), 16-20.

Bersin, J. (2004). The blended learning book: Best practices, proven methodologies, and lessons learned. San Francisco, CA: Pfeiffer.

Coaten, N. (2003). Blended e-learning. Educaweb, 69. Recuperado de http:// www.educaweb.com/esp/servicios/ monografico/formacionvirtual/11 81076. asp

Cottrell, D. M., y Robison, R. A. (2003). Blended learning in an accounting course. The Quarterly Review of Distance Education, 4(3), 261-269.

Devaney, L. (2014). Top predictions for tomorrow's classrooms. Recuperado de https://www.eschoolnews. $\mathrm{com} / 2014 / 09 / 08 /$ future-tomorrowsclassrooms-934/

Driscoll, T. (2012). Flipped Learning and democratic Education: The Complete Report. Recuperado de http://www. flipped-history.com/2012/12/flippedlearning-democratic-education.htm
García Aretio, L. (2001). Educación a distancia. De la teoría a la práctica. Barcelona: Ariel.

García Aretio, L. (2004a). Blended learning, ¿es tan innovador? Editorial $B E N E D$. Recuperado de http://e-spacio. uned.es/fez/eserv/bibliuned:20108/ blendlerninnovador.pdf

García Aretio, L. (2004b). Viejos y nuevos modelos de educación a distancia. Bordón, 56(3 y 4), 409-429.

García Aretio, L. (2009). ¿Por qué va ganando la educación a distancia? Madrid: UNED.

García Aretio, L. (2011). El fenómeno Khan. Blog García Aretio. Recuperado de http://aretio.blogspot.com.es/2011/05/ el-fenomeno-khan.htm

García Aretio, L. (2012). Convergencia presencia-distancia. Contextos universitarios mediados, 12(2). Recuperado de https://goo.gl/HYnxZp

García Aretio, L. (2014). Bases, mediaciones y futuro de la educación a distancia en la sociedad digital. Madrid: Síntesis.

García Aretio, L., y Ruíz Corbella, M. (2010). La eficacia de la educación a distancia: ¿un problema resuelto? Teoría de la educación, 22(1). ISSN: 1130-3743.

García Aretio, L., y Marín Ibáñez, R. (1998). Introducción general en Aprendizaje abierto y a distancia. Perspectivas 
y consideraciones políticas. Madrid: UNED-UNESCO.

Garrison, D. R., y Vaughan, N. D. (2008). Community of inquiry and blended learning. In Blended learning in higher education: Framework, principles, and guidelines. San Francisco, CA: JosseyBass, (pp. 13-48).

Graham, C. (2005). Handbook of blended learning: Global Perspectives, local designs. San Francisco, CA: Pfeiffer Publishing.

Gülbahar, Y., y Madran, R. (2009). Communication and collaboration, satisfaction, equity, and autonomy in blended learning environments: A case from Turkey. International Review of Research in Open and Distance Learning, $10(2), 117-138$.

Horn, M., y Staker, H. (2011). The Rise of $\mathrm{K}-12$ Blended Learning. Innosight Institute. Recuperado de https://www. christenseninstitute.org/publications/ the-rise-of-k-12-blended-learning/

Jeong, J. S., González, D., y Cañada, F. (2016). Students' Perceptions and Emotions Toward Learning in a Flipped General Science Classroom. Journal of Science Education and Technology, 25(5), 747-758.

Jen-Her, W., Robert, D.T., y Tzyh-Lih, H. (2010). A study of student satisfaction in a blended e-learning system environment. Computers \& Education, 55(1).

Lage, M. J., Platt, G. J., y Treglia, M. (2000). Inverting the Classroom: A Gateway to Creating an Inclusive Learning Environment. The Journal of Economic Education, 31(1).

Laumakis, M., Graham, C., y Dziuban, C. (2009). The Sloan-C pillars and boundary objects as a framework for evaluating blended learning. Journal for Asynchronous Learning Networks, 13(1). Recuperado de http:/ onlinelearningconsortium.org/read journal-issues,
Llorente, M. C. (2009). Formación semipresencial apoyada en Red (Blended Learning). Diseño de acciones para el aprendizaje. Eduforma, Alcalá de Guadaira.

Mantyla, K. (2001). Blending e-Learning. USA: ASTD.

Means, B., Toyama, Y., Murphy, R., Bakia, M., y Jones, K. (2009). Evaluation of Evidence-Based Practices in Online Learning: A Meta-Analysis and Review of Online Learning Studies. US Department of Education.

Niekerk, J., y Webb, P. (2016). The effectiveness of brain-compatible blended learning material in the teaching of programming logic. Computers \& Education, 103, 16-27.

Reynolds, T., y Greiner, C. (2005). Integrated field experiences in online teacher education: A natural blend? En C. J. Bonk y C. R. Graham (Eds.), Handbook of blended learning: Global perspectives, local designs. San Francisco: Pfeiffer Publishing.

Siemens, G., Gašević, D., y Dawson, S. (2015). Preparing for the digital university: a review of the history and current state of distance, blended, and online learning. Recuperado de http://linkresearchlab. org/PreparingDigitalUniversity.pdf

Singh, H. (2003). Building effective blended learning programs. Education and Technology, 43(6), 51-54.

Tourón, J., y Santiago, R. (2015). El modelo Flipped Learning y el desarrollo del talento en la escuela. Revista de Educación, 368, 196-231.

Turpo, O. (2010). Contexto y desarrollo de la modalidad educativa blended learning en el sistema universitario iberoamericano. Revista Mexicana de Investigación Educativa, 15(45), 345-370.

Usta, E., y Ozdemir, S. M. (2007). An analysis of students' opinions about blended learning environment. Paper presented at 
the International Educational Technology (IETC) Conference, Nicosia, Turkey. Vaughan, N., y Garrison, D. R. (2005). Creating cognitive presence in a blended faculty development community. Internet and Higher Education, 8(1), 1-12.

\section{Como citar este artículo:}

García Aretio, L. (2018). Blended learning y la convergencia entre la educación presencial y a distancia. RIED. Revista Iberoamericana de Educación a Distancia, 21(1), pp. 09-22. doi: http://dx.doi.org/10.5944/ried.21.1.19683 